\title{
Exploiting solar wind time series correlation with magnetospheric response by using an hybrid neuro-wavelet approach
}

\author{
Christian Napoli ${ }^{1}$, Francesco Bonanno ${ }^{2}$ and Giacomo Capizzi ${ }^{2}$ \\ ${ }^{1}$ Dept. of Physics and Astronomy, University of Catania, \\ Via S. Sofia, 95125, Catania - ITALY \\ email: chnapoli@gmail.com \\ ${ }^{2}$ Dept. of Electrical, Electronic and Systems Engineering, University of Catania, \\ Viale A. Doria, 95125, Catania - ITALY \\ email: gcapizzi@diees.unict.it
}

\begin{abstract}
The studies about the Sun rise a strong interest regarding modifications caused by the solar activity on the Earth. For almost a century in literature was discussed the problem of forecasting and analysis of the space weather, which in his definition covers both the nearearth space and the biospheric affection due to the environmental interaction with the Sun. In particular in the last years increased the attention for magnetospheric response in conjunction with the technological infrastructure and the biosphere itself. This to prevent i.e. spacecraft failures or possible treats to human health. Since the main effect of the activity of the Sun is the solar wind, rises the aim to found a correlation between itself and the localized variations induced on the magnetosphere being the purpose to predict long-term variation of the magnetic field from solar wind time series. As recently proposed for solar wind forecasting, an hybrid approach will be here used than joining the wavelet analysis with the prediction capabilities of recurrent neural networks with an adaptive amplitude activation function algorithm in order to avoid the need to standardize or rescaling the input signal and to match the exact range of the activation function.
\end{abstract}

Keywords. solar wind, magnetic fields, Sun: activity, methods: data analysis

\section{Introduction}

Modern industry, army and science operations have expanded their area of interests to the ultimate high ground of the cosmos where space based communications, navigation, scientific and surveillance systems are all affected by the operating environment. It includes the ionized turbulences in the ionopshere, which can degrade the communication signal between Earth and orbital devices and full impacts range from the energetic particles in the Van Allen radiation belts which can disrupts the satellite microelectronic, and even fluctuations in the magnetosphere induced by the solar emissions. The major availability of data of solar wind respect of magnetic field measures, such as the minor cost, even in terms of infrastructures, technological effort and site-related problems, aim to correlate the magnetospheric response with the velocity of solar wind. As recently proposed (Napoli, Bonanno \& Capizzi 2010) the problem can be faced by using hybrid computing techniques as wavelet and recurrent neural networks.

\section{The basic of neuro-wavelet theory}

Neural networks (NNs) are a promising technique to exploit the complexity of nonlinear data correlation, but the main problem in connectionist theory regards the 
development of learning algorithms that can optimize the computational capabilities of these networks. A wide variety of different approaches have been proposed during the last years, in particular a branch of research have focused on learning algorithms that use recurrent connections to deal with time series (Williams \& Zipser 1989). In a general framework for this kind of problems it is possible to unfold a recurrent neural network (RNN) into a multilayer feedforward network (FF) that grows of one layer on each time step. This approach is called backpropagation through time (BPTT). The main advantages of BPTT relies on his generality, then is possible to improve the predictive capability and reduce the training time of this kind of RNNs using adaptive amplitude of activation functions based on a real time recurrent algorithm (AARTRL) which can be employed as nonlinear adaptive filter for fully connected RNNs. The inputs for the RNN were given by the set of coefficients coming from the biorthogonal wavelet decomposition of the solar wind velocity time series. Wavelet analysis was used in order to reduce the data redundancies so obtaining representation which can express their intrinsic structure. In fact the main advantage of the use of wavelet is the ability to pack the energy of a signal, and then the relevant informations carried by it, in few significant uncoupled coefficients. This characteristic is very useful to optimize the performances of neural networks (M. M. Gupta, L. Jin, N. Homma 2003). As recently proposed a biorthogonal wavelet decomposition was used due to his freedom degrees. The biorthogonal wavelets utilize a particular transform, which is invertible but not necessarily orthogonal, based on coupled filters, and allowing more degrees of freedom respect to traditional orthogonal (and of course the not orthogonal) wavelets (S. Mallat 2009).

\section{Proposed data analysis}

In this paper the authors propose hybrid fully recurrent neuro-wavelet RNNs, to correlate the magnetopsheric response to the solar wind and forecast these variations from predicted solar wind data. To obtain a correct prediction of magnetic field from solar wind velocity data an RNN was designed starting on the data series. The inputs for the RNN were given by the set of coefficients coming from the biorthogonal wavelet decomposition of the solar wind velocity time series. By using the MATLAB package we developed a series of routines performing a biorthogonal wavelet analysis of the data of solar wind and magnetic field extract a shortened number of non-zero coefficients from a representative time series. This method allowed to drastically reduce the coefficients data sets aiming to correlate them. The subbands of the decomposition of solar wind data then were used to predict the future values of wavelet coefficients for the magnetic field. As advanced features of the process the temporal trend was associated with the different sub-bands of the decomposition, allowing to subdivide the neural network in modular serial subnets in order to gain computation time and drop down the complexity. The available data, regarding a time period from january 1998 to may 2010, were collected during the WIND mission of the NASA administration. WIND is a spin stabilized spacecraft launched in November 1, 1994 and placed in a halo orbit around the L1 Lagrange point, more than 200 Re upstream of Earth to observe the unperturbed solar wind that is about to impact the magnetosphere of Earth. The solar wind data are provided, as done in a previous paper by the SWE. While the magnetic field data are provided by the Magnetic Field Investigation, a subsystem consistent of dual triaxial fluxgate magnetometers on a $12 \mathrm{~m}$ radial boom, designed to provide accurate, high resolution vector magnetic field measurements in near real time on a continuous basis in a wide dynamic measuring range. A detailed description of the experimental system can be found in the (PhD dissertation of J. Kasper 2002). The solar wind data were deceomposed as reported in (Napoli, Bonanno 


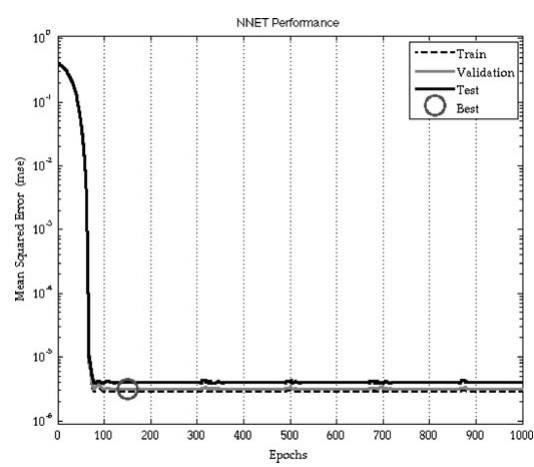

Figure 1. Performance of the NN

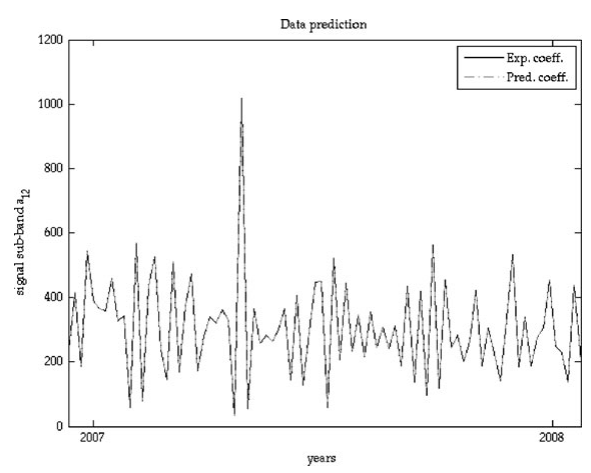

Figure 2. Magnetic field prediction

\& Capizzi 2010), while the magnetic field data were also decomposed in 13 subbands with the described method reducing the $99.75 \%$ of the energy in $0.05 \%$ of non zero coefficients. We trained the network to the 12th temporal sub-band to predict the rate of change in the magnetic field for the next 6 days by using solar wind data. For a magnetic field time sub band $b_{n}$, and in order to predict the data at a time interval $\tau_{1}$ from a time serie of a previous interval $\tau_{0}$, then the input for the $\mathrm{NN}$ is given in the form of solar wind time series $\left[a_{n+1}\left(\tau_{0}\right) \mid d_{n}\left(\tau_{0}\right)\right]$ so that the output will be the sub band $b_{n}\left(\tau_{1}\right)$. The NN was a full RNN NARX, trained by a gradient descent with momentum and adaptive amplitude learning rule backpropagation algorithm and composed by 3 hidden layer: the first of 13 neuron with tribas transfer function (tf), the second of 7 neurons with tansig tf, and the third of 5 neurons with a competitive tf. Finally time vectors were used to create 5 delay lines for the input and 3 for the output.

\section{Conclusions and results}

The simulation results show the power and the good performance obtained with the proposed method. The predicted magnetic field accurately matches the experimental data within an RMS of less than $0.4 \%$ as shown in fig. 1 and 2 . The high degree of accuracy highlights the correlation between magnetic field measurements in the L1 point and solar activity in the form of solar wind creating the basis for further works about prediction of solar manifestations (such coronal holes or flares) by mapping the magnetic field near the Earth orbit.

\section{References}

Napoli C. et al. 2010, in: Advances in Plasma Astrophysics, Proc. IAU Symposium No. 274 Capizzi G., Bonanno F., \& Napoli C. 2010, Proc. Speedam 2010, p. 586

Williams R. J. \& Zipser D. 1989, Neural Comput., Vol.1, p. 270

M. M. Gupta et al. 2003, Static and Dynamic Neural Networks, J. Wiley \& Sons Inc. S. Mallat 2009, A Wavelet Tour of Signal Processing: The Sparse Way, Academic Press Kasper J. 2002, Excerpts from PhD dissertation, Cap.2, p. 49

Gleisner H., Lundstedt H., \& Wintoft P. 1996, Ann. Geophys., Vol.4, No.7, p. 679

Eselevich V. G. et al. 2009, Cosmic Res., Vol.47, No.2, p. 95 